\title{
A SIMPLE APPROACH TO THE ANALYTIC CONTINUATION AND VALUES AT NEGATIVE INTEGERS FOR RIEMANN'S ZETA-FUNCTION
}

\author{
DAVID GOSS
}

\begin{abstract}
In this paper, the author presents a new approach to the subjects in the title, putting them in a new light. In fact, only integration by parts is used. This approach has two advantages: (1) it makes the p-adic theory seem even more natural, and (2) it is accessible to readers with only one year of basic calculus, making the subjects reachable in elementary courses.
\end{abstract}

Many of the deepest properties of the Riemann zeta-function appear (with or without proof) in the work of Leonhard Euler. Euler's methods (which are summarized quite nicely in [1]) are extremely arithmetic in that they involve power series manipulations. As such, they are perfect for $p$-adic use (see N. Katz [4]). Euler's goal was to compute $\zeta(n)$ for $n \in Z-\{1\}$. In this he succeeded in finding a closed form solution for all except the positive odd integers, a still open problem.

It seems the first person to seriously consider $\zeta(s)$ for all positive reals was Dirichlet, though Euler certainly was in a position to do so had he chosen. It was Riemann who finally made the crucial step of considering $\zeta(s), s$ complex, in order to describe $\zeta(s)$ by its zeroes.

Euler computed $\zeta(-k)$ by using Abel summation in a very clever way; in fact he anticipated Abel by many years. This was first made rigorous by Riemann through complex methods. Here we present an elementary approach to these values that is rigorous by modern standards, yet could have been used by Euler as further justification for his computations. In doing so we obtain a simple means of analytic continuation for $\zeta(s)$ as well as a variety of integral formulas. In fact, we use only integration by parts. In light of this method, the $p$-adic theory becomes even more natural and seems almost forced.

The author thanks Mike Rosen and Steve Galovich for their encouragement.

We begin by recalling Euler's integral for $n$ ! (see [2]),

$$
n !=\int_{0}^{1}(-\log x)^{n} d x
$$

Legendre set $x=e^{-t}$ to obtain

$$
n !=\int_{0}^{\infty} t^{n} e^{-t} d t
$$

Received by the editors April 11, 1980 and, in revised form, August 27, 1980. 1980 Mathematics Subject Classification. Primary 10H05; Secondary 10 H08. 
and he set for $s>0$

$$
\Gamma(s)=\int_{0}^{\infty} e^{-t} t^{s-1} d t, \quad \Gamma(n+1)=n ! .
$$

This integral makes sense for all $s \in \mathbf{C}$ with $\mathbf{R} e(s)>0$, and defines a holomorphic function there.

Now, we may extend $\Gamma(s)$ to all $s \in \mathbf{R}$ (or $\mathbf{C}$ ), in the following well-known, simple fashion: Apply integration by parts to (1) to obtain

$$
\Gamma(s)=\left.\frac{t^{s}}{s e^{-t}}\right|_{0} ^{\infty}+\frac{1}{s} \int_{0}^{\infty} t^{s} e^{-t} d t=\frac{1}{s} \int_{0}^{\infty} t^{s} e^{-t} d t .
$$

But this new integral makes sense for all $s>-1(\operatorname{Re}(s)>-1)$; thus extending $\Gamma(s)$ meromorphically to this region. Integration by parts then extends $\Gamma(s)$ to all $s$ with $s>-2$, etc. By continuing we cover the whole plane. As a bonus, we obtain $s \Gamma(s)=\Gamma(s+1)$.

By analytic continuation the above extension is the extension. Henceforth, we suppress the complex point of view.

Let $\zeta(s)=\sum_{n=1}^{\infty} n^{-s}$ be Riemann's zeta-function. By the standard integral test this converges for all $s \in \mathbf{R}, s>1$. Euler and Dirichlet proved that

$$
\zeta(s)=\prod_{p \text { prime }}\left(1-p^{-s}\right)^{-1}, \quad s>1 .
$$

It was noticed by Abel for $s \in \mathbf{N}^{+}-\{1\}$ and Riemann for all $s>1$ that $\Gamma(s) \zeta(s)$ may be given the following simple integral form: As $\Gamma(s) n^{-s}=\int_{0}^{\infty} t^{s-1} e^{-n t} d t$, one has

$$
\Gamma(s) \zeta(s)=\int_{0}^{\infty} t^{s-1}\left(\sum_{n=1}^{\infty} e^{-n t}\right) d t=\int_{0}^{\infty} t^{s-1} \frac{e^{-t}}{1-e^{-t}} d t .
$$

We modify this à la Euler (and Katz) by considering the functions for $m \in \mathbf{N}^{+}$,

$$
\phi_{m}(s)=\left(1-m^{1-s}\right) \zeta(s), \quad s>1 .
$$

However, it is immediate that $\phi_{m}(s)$ converges for all $s>0$ by Leibniz's test. In the fashion just given it is easy to see that for $s>0$,

$$
\Gamma(s) \phi_{m}(s)=\int_{0}^{\infty} t^{s-1} R_{m}\left(e^{-t}\right) d t
$$

where

$$
\begin{aligned}
R_{m}(z) & =\frac{z+z^{2}+\cdots+z^{m-1}+(1-m) z^{m}}{1-z^{m}} \\
& =z \frac{1+z+\cdots+z^{m-2}+(1-m) z^{m-1}}{1-z^{m}} .
\end{aligned}
$$

As the numerator and denominator of $R_{m}$ are 0 at 1 , we may write

$$
R_{m}(z)=\frac{z N_{m}(z)}{1+z+\cdots+z^{m-1}}, \quad N_{m}(z)=\sum_{k=1}^{m-1} k z^{k-1} .
$$

Note that $R_{m}(0)=0$. For example, $R_{2}(z)=z /(1+z)$. 
Therefore, as $t \rightarrow 0, R_{m}\left(e^{-t}\right)$ remains bounded; and so we can apply integration by parts to find

$$
\begin{aligned}
\phi_{m}(s) & =\left.\frac{1}{s \Gamma(s)} t^{s} R_{m}\left(e^{-t}\right)\right|_{0} ^{\infty}-\frac{1}{s \Gamma(s)} \int_{0}^{\infty} t^{s} R_{m}\left(e^{-t}\right)^{\prime} d t \\
& =\frac{1}{\Gamma(s+1)} \int_{0}^{\infty} t^{s} R_{m}\left(1, e^{-t}\right) d t
\end{aligned}
$$

where $R_{m}\left(1, e^{-t}\right)=R_{m}\left(e^{-t}\right)^{\prime}$. However, this integral converges for all $s$ with $s>-1$. As we have previously extended $\Gamma(s)$ to all $s$, we now can extend $\phi_{m}(s)$ and $\zeta(s)$ to all $s$ with $s>-1$. Moreover, $R_{m}\left(1, e^{-t}\right)$ also remains bounded and so we can again apply the process. Continuing in this fashion, we extend $\phi_{m}(s)$ and $\zeta(s)$ to all $s \in \mathbf{R}$.

As a conequence we obtain the following: Let $s \in \mathbf{R}$ and let $j \in \mathbf{N}$ with $j>\operatorname{Min}(-s,|s|)$. Then

$$
\phi_{m}(s)=\frac{1}{\Gamma(s+j)} \int_{0}^{\infty} t^{s+j-1} R_{m}\left(j, e^{-t}\right) d t,
$$

where

$$
R_{m}(j, z)=\left(z \frac{d}{d z}\right)^{j} R_{m}(z)
$$

Next we turn to evaluating $\phi_{m}(-k)$ for $k \in \mathbf{N}$. We show $\phi_{m}(-k)=R_{m}(k, 1)$, which will then be equated with Bernoulli numbers, and is Euler's original computation in terms of Abel summation when $\boldsymbol{m}=2$.

Set $j=k+1$ in our formulas above. We find

$$
\begin{aligned}
\phi_{m}(-k) & =\frac{1}{\Gamma(1)} \int_{0}^{\infty} R_{m}\left(k+1, e^{-t}\right) d t \\
& =\int_{0}^{\infty} R_{m}\left(k+1, e^{-t}\right) d t
\end{aligned}
$$

Now set $t=-\log y$ to find

$$
\begin{aligned}
\phi_{m}(-k) & =\int_{0}^{1} \frac{R_{m}(k+1, y) d y}{y}=\int_{0}^{1} \frac{y \frac{d}{d y} R_{m}(k, y) d y}{y} \\
& =\int_{0}^{1} \frac{d}{d y} R_{m}(k, y) d y=R_{m}(k, 1)-R_{m}(k, 0) \\
& =R_{m}(k, 1), \text { as } R_{m}(k, 0)=0 .
\end{aligned}
$$

Definition. The Bernoulli numbers $\left\{B_{k}\right\}$ are defined by the power series

$$
F(t)=\frac{t}{e^{t}-1}=\sum_{k=0}^{\infty} B_{k} t^{k} / k ! .
$$

As $F(-t)-F(t)=t$, we see

$$
B_{k}=0, \quad k=2 n+1, n>0 .
$$


Now,

$$
R_{m}(k, 1)=\lim _{z \rightarrow 1}\left(z \frac{d}{d z}\right)^{k}\left(\frac{z+z^{2}+\cdots+z^{m-1}+(1-m) z^{m}}{1-z^{m}}\right) .
$$

If we set $z=e^{t}$, then $z(d / d z)=d / d t$ and

$$
\begin{aligned}
R_{m}(k, 1) & =\lim _{t \rightarrow 0}\left(\frac{d}{d t}\right)^{k}\left(\frac{e^{t}+\cdots+e^{(m-1) t}+(1-m) e^{m t}}{1-e^{m t}}\right) \\
& =\lim _{t \rightarrow 0}\left(\frac{d}{d t}\right)^{k}\left(\frac{e^{t}}{1-e^{m t}} \cdot\left(\frac{1-e^{m t}}{1-e^{t}}-m e^{(m-1) t}\right)\right) \\
& =\lim _{t \rightarrow 0}\left(\frac{d}{d t}\right)^{k}\left(\frac{e^{t}}{1-e^{t}}-\frac{m e^{m t}}{1-e^{m t}}\right) \\
& =\lim _{t \rightarrow 0}\left(\frac{d}{d t}\right)^{k}\left(\frac{1}{e^{-t}-1}-\frac{m}{e^{-m t}-1}\right) \\
& =\lim _{t \rightarrow 0}\left(\frac{d}{d t}\right)^{k}\left(\sum_{j=0}^{\infty}(-1)^{j-1} \frac{B_{j} t^{j-1}}{j !}-m \sum_{j=0}^{\infty}(-m)^{j-1} \frac{B_{j} t^{j-1}}{j !}\right) \\
& =(-1)^{k} \frac{B_{k+1}}{k+1}\left(1-m^{k+1}\right) .
\end{aligned}
$$

Thus $R_{m}(0,1)=-\frac{1}{2}(1-m)$. But, if $k$ is even $>0$, then $B_{k+1}=0$. So, for $k>0$

$$
\phi_{m}(-k)=R_{m}(k, 1)=\frac{-B_{k+1}}{k+1}\left(1-m^{k+1}\right),
$$

and so $\zeta(-k)=-B_{k+1} /(k+1)$. This is the standard form for the value.

Finally, we make some remarks of a less elementary nature. The first is that our methods work also for the partial zeta-functions, i.e., those functions of the form

$$
\sum_{n=1}^{\infty}(n+a)^{-s}
$$

for $0<a<1$.

The second is that the functions $R_{m}(j, z)$ are precisely those that occur $p$-adically, see [4]. In fact, the whole $p$-adic theory is codified by $R_{m}(z)$ in that it "is" the $p$-adic measure, when $(p, m)=1$.

Lastly, Mellin-inversion (see [3]) gives us a means to invert our formulas so as to obtain the functions $R_{m}(k, z)$ as integrals involving the gamma and zeta functions. Using it, we find

$$
\phi_{m}(-k)=R_{m}(k, 1)=\lim _{x \rightarrow 0^{+}} \int_{\sigma-i^{\infty}}^{\sigma+i^{\infty}} \Gamma(s) \phi_{m}(s-k) x^{-s} d s,
$$

for any $\sigma>0$. 


\section{REFERENCES}

1. R. Ayoub, Euler and the zeta-function, Amer. Math. Monthly 81 (1974), 1067-1086.

2. P. Davis, Leonhard Euler's integral: A historical profile of the gamma function, Amer. Math. Monthly 66 (1959), 849-869.

3. H. M Edwards, Riemann's zeta-function, Academic Press, New York, 1974.

4. N. Katz, P-adic L-functions via moduli of elliptic curves, Proc. Sympos. Pure Math., vol. 29, Amer. Math. Soc., Providence, R. I., 1975, pp. 479-506.

Department of Mathematics, University of California at Berkeley, Berkaley, Califormi 94720 